\title{
Administration of DNA Plasmid Coding Protein Aggregating Domain Induces Inflammatory Bone Loss
}

\author{
Dimitrios Agas ${ }^{1}$, Fabio Concetti ${ }^{1}$, Melania Capitani ${ }^{1}$, Giovanna Lacava ${ }^{1}$, Antonio Concetti ${ }^{1}$, \\ Luigi Marchetti ${ }^{1}$, Fulvio Laus ${ }^{1}$, Andrea Marchegiani ${ }^{1}$, Vasco Azevedo ${ }^{2}$, Maria Giovanna Sabbieti ${ }^{1, *, \#}$ \\ and Franco Maria Venanzi ${ }^{1, *, \#}$
}

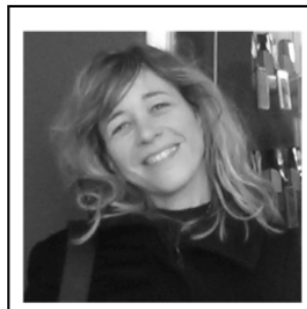

M.G. Sabbieti

${ }^{I}$ School of Biosciences \& Veterinary Medicine, University of Camerino, 62032 Camerino (MC), Italy; ${ }^{2}$ Laboratory of Cellular and Molecular Genetics (LGCM), Department of General Biology, ICB/UFMG. Belo Horizonte, Minas Gerais, Brazil

Abstract: $\underline{\text { Background: }}$ Plasmids coding protein aggregation polypeptides from different sources have been proposed as genetic adjuvants for DNA vaccines. We reported that a plasmid (pATRex), encompassing the DNA sequence for the von Willebrand A (vWA/A) domain of the Anthrax Toxin Receptor-1 (ANTXR-1, alias TEM8, Tumor Endothelial Marker 8), acts as strong immune adjuvant by inducing formation of in-

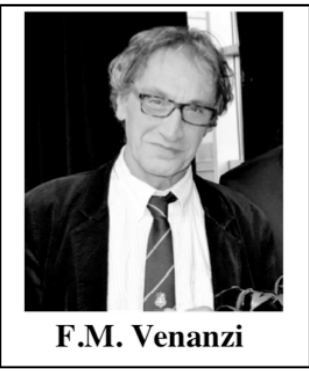
soluble intracellular aggregates and subsequent cell death. Objective: In the present study we addressed the question of whether there is any substantial immunotoxicity associated with the use of self-aggregating proteins as genetic adjuvants. Methods \& Results: Here we report, by mean of histology, X-ray and molecular examinations of bone specimens, the unexpected finding that intramuscular injection of pATRex in mice triggers, per se, severe bone loss (osteoporosis) independently from the sex and genotype of the treated animals. Conclusion: Even though the study suggests that proteinaceous "sticky " adjuvants are unlikely to find their way into practical vaccination, the information gained is of value as ATRex injections could provide an additional, simplified, mouse model of osteoporosis. Moreover, our results provide experimental support to the hypothesis that proteotoxic aggregates chronically activate the innate immune system in amyloid and aggregosome associated disorders.

Keywords: DNA vaccines, Adjuvants, Protein aggregates, Chronic inflammation, Bone remodeling, Osteoporosis.

\section{INTRODUCTION}

DNA vaccination delivers plasmid DNA encoding the target gene to induce both humoral and cellular immune responses. This strategy has been used for more than two decades to treat autoimmune disease, viral infection and cancer [1]. However, while DNA vaccines have reached clinical setting, their use has been in general limited because of their restricted ability to induce strong immune responses in higher primate and humans [2]. Therefore, DNA vaccines will require a new generation of adjuvants for optimal efficacy. However, in any case, one of the major challenges in adjuvant research is to gain potency while minimizing toxicity [3].

It has been reported that the fusion of antigen-coding DNA with genic sequences for protein's aggregationpromoting domains from different sources, may enhance the antigenic potential of DNA vaccines $[4,5]$. In this frame we recently demonstrated that a plasmid vector (pATRex)

\footnotetext{
*Address correspondence to these authors at the School of Biosciences \& Veterinary Medicine, University of Camerino, Camerino (MC) Italy; Tel: +39-0737-403258; Facsimile: +39-0737- 403209;

E-mails: giovanna.sabbieti@unicam.it; franco.venanzi@unicam.it

\# These authors contributed equally as senior authors.
}

encompassing the DNA sequence for the von Willebrand I / A domain (VWA/A) of Anthrax Toxin Receptor-1 (ANTXR1, alias Tumor Endothelial Marker 8- TEM8), when coadministered with different DNA vaccines enhanced immune protection against tumors (e.g. Breast cancer, Melanoma) and infectious disease (e.g. Malaria) [6,7]. Further, we got evidence that pATRex immune-adjuvancy was related to its ability to trigger insoluble intracellular aggregates (aggregosomes) ( ${ }^{*}$ see foot note), caspases activation (e.g. caspases \# 9, \# 3 and \# 12), and apoptotic cell death, leading in turn to the stimulation of the innate immune system [7]. In agreement with the notion that aggregosomes preferentially activate the innate immune system, we found that pATRex injections, without any joint-specific antigen, has no effect on the acquired immunosystem as neither B or T cells ATRex-

\footnotetext{
* $v W A / A$ domain, the essential core of pATRex sequence, is a widely distributed structural module in cell-matrix adhesive proteins, reported to selfaggregate, and to be involved in multiprotein complexes formation (Whittaker CA, Hynes RO. Distribution and evolution of von Willebrand /Integrin domains: widely dispersed domain with role in cell adhesions and elsewhere. Mol Biol Cell.2002; 13: 3369-87)
} 
specfic immunity could be detected after plasmid administration [6, and unpublished results.]

An important question to be addressed is whether there are any safety issues posed by the use of self-aggregating proteins as genetic adjuvants. Could the release of pATRex aggregates by dying cells trigger protein aggregation in adjacent cells such as occur in prion disease, amyloid and otheraggregosome disorders? We can also formulate the questions of whether the induction of cell death at the injection site or wherever else the plasmid distributes in the body may result in immune toxicity. The concerns are justified as an association between inflammatory disorders and altered protein homeostasis (proteostasis) is now widely recognized. Indeed, on one side stands the observation that the products of inflammation might impair proteasomes activity leading to altered proteostasis and subsequent proteins aggregation $[8$, 9]. On the other side, the possibility that aggregates may induce inflammation is supported by a plethora of experimental data [10-12]. In this scenario it was hypothesized that the persistence of aggregosomes in vivo could initiate a self reinforcing cycle of pro-inflammatory signals, ending up in chronic inflammation that predisposes the organism to develop a host of systemic diseases (i.e. obstructive pulmonary disease, insulin resistance, Paget's disease of bone, cancer, and neurodegenerative diseases) [13].

Although our previous studies excluded substantial histopathological changes in a wide array of soft tissues following pATRex DNA administration [6], we extended our survey to bones. The choice of bone as read-out system for immunotoxiciy (a neglected target tissue in studies about the reactogenicity of vaccine adjuvants) is based on several considerations: i) Bone is the main supplier of immunocells to inflamed tissues, and its exquisitely sensitive to inflammation [14]. ii) Bone homeostasis is dinamically influenced by the immune system, with lymphocytes and macrophages being key mediators of the osteo-immune regulation [15]. As complex and intertwisted, these interactions are ultimately mediated through the effects of bone remodeling, a coordinate process between formation and degradation of the bone respectively managed by osteoblasts (OBs) and osteoclast (OCs), essentially dictated by a fine-tuned equilibrium between the pro and anti-inflammatory conditions [16]. iii) Wherever chronic inflammatory diseases occurs, systemic effect on bone will ensue in bone loss (e.g. osteoporosis) [17]. In example, epidemiological studies highlight an association between neurological disorders caused by protein aggregates and an increased risk of developing osteoporosis: Parkinson Disease [18-20], Huntington Diseases [21-23], and Alzheimer Disease [24, 25]. Notably, amyloid $\beta$ peptides from Alzheimer's patients have been reported to accumulate in osteoporotic bone tissue and to enhance osteoclast function [26]. iv) Finally, relevant to our work, there are reports about the ability of i.m injected plasmids to reach the bone marrow, and to be acquired by resident immunocells (i.e. neutrophils or monocytes) $[27,28]$.

Here we report that the delivery of pATRex in healthy mice, by uncoupling bone formation from bone resorption leads to a fast and severe inflammatory bone loss.

\section{MATERIALS \& METHODS}

\section{DNA Plasmids}

For DNA immunizations, large scale preparation of the plasmids was routinely performed by alkaline lysis using Qiagen Plasmid Maxi kits (Qiagen). To assure endotoxin free products, DNA plasmids were purified using either Endo Free Plasmid Kit (Qiagen) or Gen Elute HPSelect Plasmid Giga Prep columns (Sigma \# NA0800).

\section{Animals Trials}

Female and male FVB and Balb/c mice (Harlan Italy SrL, Correzzana Milano, Italy) were used. Mice were kept in laminar-flow cage in a standardized environmental condition. Mice (6-8 weeks old) were randomized in four subgroups (six mice for each subgroup) and injected intramuscularly (i.m.) into the hind limb at week $0,1,2$. Each mouse received at a time $100 \mu \mathrm{g}$ of DNA $(1 \mathrm{mg} / \mathrm{ml})$ in saline using an insulin syringe; the subgroups were as follows: subgroup 1: $100 \mu \mathrm{g}$ pcDNA3.1- ATRex (experimental); subgroup 2: $100 \mu \mathrm{g}$ pANTXR-1 (control, coding for the parental ANTXR- 1, alias TEM8) subgroup 3: $100 \mu \mathrm{g}$ pcDNA3.1 (scaffold plasmid); subgroup 4: only saline. At the end of these injection cycles, mice from all groups were sacrificed at $90^{\text {th }}$ days after the first injection to monitor the impact of plasmids on bone homeostasis. All mice were sacrificed by $\mathrm{CO} 2$ narcosis according to the recommendation of the Italian Ethical Committee and under the supervision of authorized investigators. To evaluate the systemic effects of the injection sites, plasmids were injected either into the left or right quadriceps, and counter-lateral bone collected.

\section{Histological Examination}

Femurs, dissected from adhering tissue, were fixed in $4 \%$ paraformaldehyde (PFA) and decalcified as previously described [29]. Samples, were embedded with Tissue-Tek OCT compound (Sakura). Then, $12 \mu \mathrm{m}$ thick sections of femurs were obtained by a rotatory $-30^{\circ} \mathrm{C}$ microtome cryostat (Ames Cryostast Miles), air-dried and stained with toluidine blue or hemotoxylin-eosin. Digital-assisted bone immage analysis was performed as previosely described [30]. Briefly, a black and white image mask was constructed. A region of interest was selected from a Toluidine blu-stained section. This image was then Adobe Photoshop ${ }^{\mathrm{TM}}$ to prepare the black-andwhite mask. Within the selected region of interest, trabecular bone was identified and represented in black.

Osteoclasts were stained by Acid Phosphatase, Leukocyte (TRAP) kit (Sigma-Aldrich, Milano, Italy) according to the manufacturer's instructions. Quantification of the TRAPstained area per $0.3 \times 0.3 \mathrm{~mm}^{2}$ under the growth plate of the proximal femur was performed using NIH ImageJ as previously described [31].

Other sections were stained with freshly prepared Oil Red O. Sections Images were captured by a Leica DM 2500 optical microscope.

\section{X-ray Analysis}

After anesthesia with isoflurane-air mixture, mice were positioned in dorsal recumbence, making sure that pelvis, femurs and tibias were included in radiographs. A portable 
X-ray generator (Gierth HF 80/15 plus ULTRA LEICH, Gierth X-Ray International $\mathrm{GmbH}$, Germany) mounted on a stative with focal distance of $60 \mathrm{~cm}$ was used; X-ray applied dose was $54 \mathrm{Kv}$ for a time of $0.04 \mathrm{sec}$. Radiographs were acquired in DICOM format with Fujifilm FCR Capsule X (Fujifilm Corporation, Japan) and processed both with Osirix (Pixmeo SARL, Switzerland) and ImageJ (http:// rsb.info.nih.gov/ij/) software, according to image analysis protocols previously reported $[32,33]$. Long bones were first processed in Osirix for region of interest (ROI) identification; afterward, the DICOM images were converted with ImageJ into TIFF images and a 16 intervals pseudo-color scale was applied to the grey scale. This scale starts from black pixels (value of zero) and increasing gradations of mineralization density are represented in 16 equal intervals by a pseudo-color scheme to white pixels (value of 255). Hence, distribution of pixels in a ROI was calculated and displayed as a histogram.

\section{Western Blotting}

Long bones (femurs, tibiae and humeri) from the above mouse groups were dissected free of adhering tissue. The ends were removed, and the marrow cavity was flushed with DMEM. Western blotting was performed as previously described [34]. Membranes were incubated with the following primary antibodies diluted in blocking buffer: rabbit antiOsteoprotegerin and mouse anti-RANKL antibodies (Abcam, Prodotti Gianni, Milano, Italy) diluted respectively 1:500 and 1:250; rabbit $\mathrm{mAb}$ anti-Runx-2 antibody (Cell Signaling, Euroclone, Milano, Italy) diluted 1:800; rabbit anti-Osterix antibody (Santa Cruz Biotechnology, Italy) diluted 1:600, rabbit anti-NF-kB and rabbit anti-TNF-a diluted 1:500 (BioLegend, Microtech SrL, Napoli, Italy). After washing blots were incubated with horseradish peroxidase (HRP)conjugated donkey anti-rabbit IgG or with HRP-conjugated rabbit anti-mouse IgG (Cell Signaling, Euroclone Milano, Italy) both diluted 1:50,000 in blocking solution for $1 \mathrm{~h}$ at RT. To normalize the bands, filters were stripped and reprobed with a monoclonal anti- $\alpha$-tubulin (Sigma-Aldrich, Milano, Italy). Bands density was quantified by densitometric scan.

\section{Real Time PCR}

Total RNA was extracted in TRIZOL reagent (Invitrogen Life Technologies, CA) from mice bone marrow obtained as above described. cDNA was synthesized using RevertAid H Minus First Strand cDNA Synthesis Kit (Thermo Scientific, MA) according to manufacturer's protocol. PCR primers are listed as follows:

collIFW, 5'-GGAAGAGCGGAGAGTACTGGATC-3' and collIRev, 5'-TACTCGAACGGGAATCCATCGGT-3' ; OCNFW, 5'-TCTGACCTCACAGATGCCAAGCCC-3' and OCNRev, 5'-TAGGCGGTCTTCAAGCCATACTGG-3'; MOUSEactinFW, 5'-TTCGTTGCCGGTCCACA-3' and MOUSEactinRev, 5'-ACCAGCGCAGCGATATCG-3'.

Real time PCR was performed in three replicates of each sample using DyNAmoTM Flash SYBR Green ${ }^{\circledR}$ qPCR Kit (Thermo Scientific, MA) on a Stratagene Mx3000P; each reaction tube contained a total of $50 \mathrm{ng}$ of cDNA into $20 \mu \mathrm{l}$ of total reaction volume. Actin gene expression was used as internal control to normalize the amount of target cDNA added to the reactions. Data are expressed as fold change of gene expression relative to empty vector injected mice.

\section{Statistical Analysis}

GraphPad Prism 5.0 for Macintosh was used for drawing graphs and for statistical analysis

(GraphPad Software, San Diego, CA, USA). All in vitro and in vivo experiments were repeated at least three times. tstudent test was used to test for significant differences $(\mathrm{p}<0.05)$ between two groups.

\section{RESULTS \& DISCUSSION}

The inflammatory potential (reactogenicity) of vaccine adjuvants on bone structure / homeostasis has been never documented. In this study we evaluated the impact of pATRex DNA administration on bone physiological remodeling. In our experiments, mice were i.m. injected for three weeks (once a week) either with pATRex or control plasmids (pANTRX-1 or sham pcDNA 3.1 vector), and sacrificed three months after the first injection (see Material \& Methods section for details). Afterwards bones were isolated and routinely analyzed for osteo-toxicity by standard techniques.

What follow are representative results as obtained from independent trials (three for each selected plasmid) in female $\mathrm{Balb} / \mathrm{c}$ mice. Notably, the results were consistently reproduced in male Balb/c, and in FVB/N mice strain of both sexes (Data available on request).

\section{Inflammatory Bone Loss: Histological, X-ray \& Molecu- lar Evidences}

Femurs from plasmids injected mice were collected for a first histological evaluation. As shown in Fig. (1) mid diaphysis femurs from pATRex-treated mice evidenced, at variance of control ANTXR1 plasmid treated mice, a dramatic loss of cortical bone resulting in deep and extended cavities suggestive of an enhanced bone resorpting activity. Moreover the analysis of the metaphyseal regions of the distal femurs also evidenced bone erosion and thinned disconnected trabeculae Fig. (2A), whose area was significantly reduced as assessed by digital-assisted bone image analysis [30] (Fig. 2B). Even though that these basic histological observations, per se, do not allow an accurate quantitative analysis of trabecular and cortical changes, as obtained by using either software-assisted histomorphometry or X-ray microtomographic $(\mu \mathrm{CT})$, they high reproducibility over a large number of sections and animals examined decidedly demonstrates that pATRex injections selectively induce bone resorption.

Additionally, we got evidence that pATRex administration significantly deteriorated bone mineral and organic matrices (bone density). Indeed, while X-ray scanning evidenced an important demineralization (approx. $25 \%$ compared to control ANTXR1 plasmid treated mice) Fig. (3 A, B), quantitative real time-RT PCR analysis demonstrate a down-regulation of both Collagen I (that accounts for approx. $90 \%$ of organic matrix) and osteocalcin mRNAs expression, the latter been considered an important marker of bone formation (Fig. 3C) [35]. 


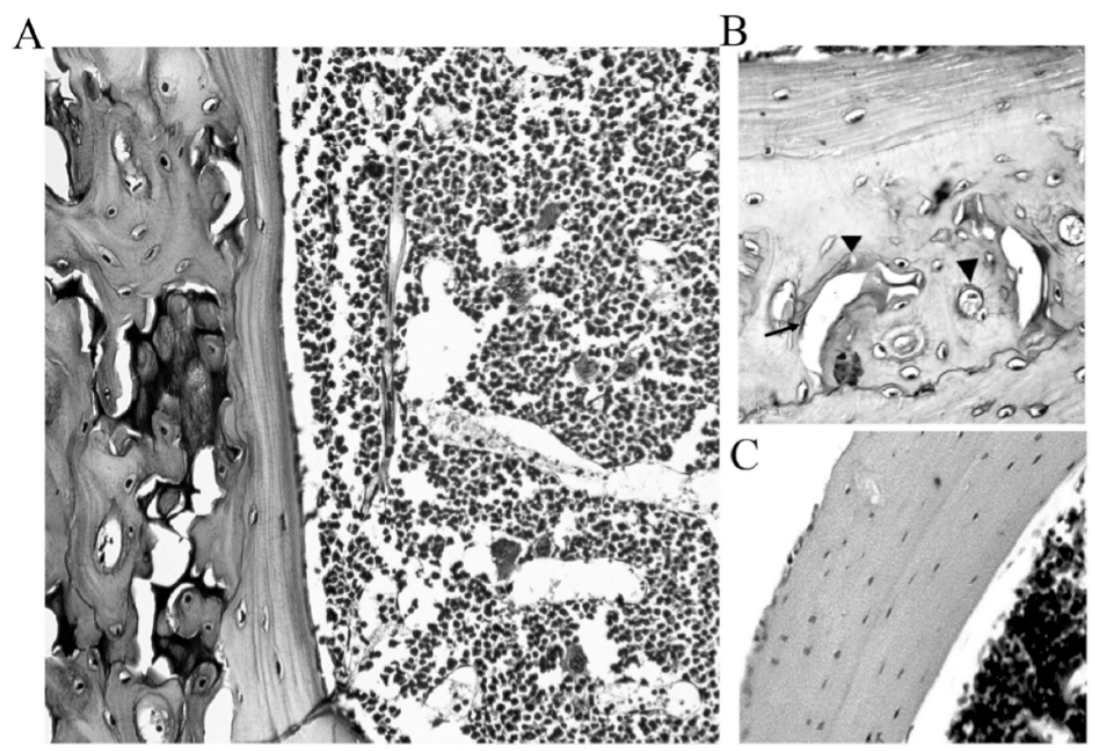

Fig. (1). Representative sections toluidine bleu stained of femur mid diaphyses from pATRex DNA (A, B) and ANTXR1 DNA-injected mice (C). Arrows indicate resorption cavities within the cortex, and arrows head point to osteoclastic activity. Magnifications: 40x (A, B); 20x (C). Number of mice examined $(\mathrm{N})=7$ per group.

A

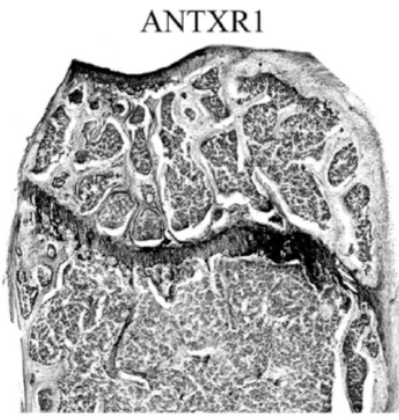

ANTXR1

B

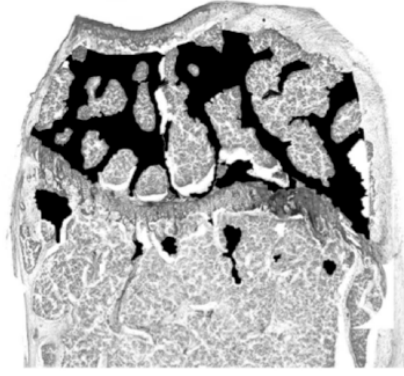

pATRex

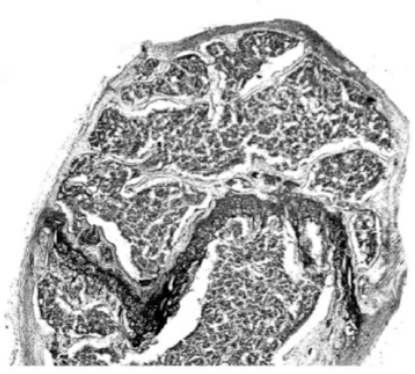

pATRex

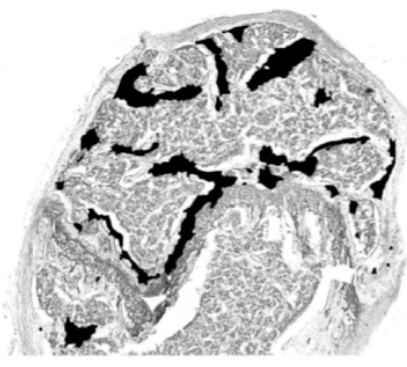

\section{Trabecular Bone area $\left(\mathrm{mm}^{2}\right)$}

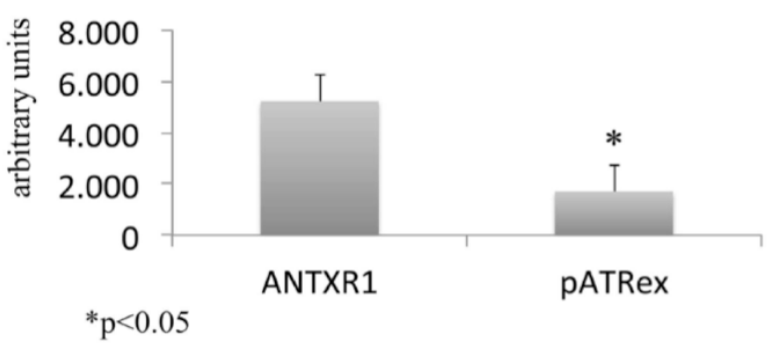

Fig. (2). Representative reconstruction toluidine bleu stained of metaphyseal regions of distal femurs from ANTXR1 DNA and pATRex DNA-injected mice (A). Representative reconstructions opened in Adobe Photoshop ${ }^{\mathbb{B}}$ to prepare the black and white mask. Bone was identified and represented in black. Trabecular bone area quantification was performed by Image $\mathrm{J}$ software. Statistical analysis derives from six independent measurements of the trabecular bone area $(\mathbf{B}) ;{ }^{*} p<0.05 \mathrm{~N}=7$ per group. 
A
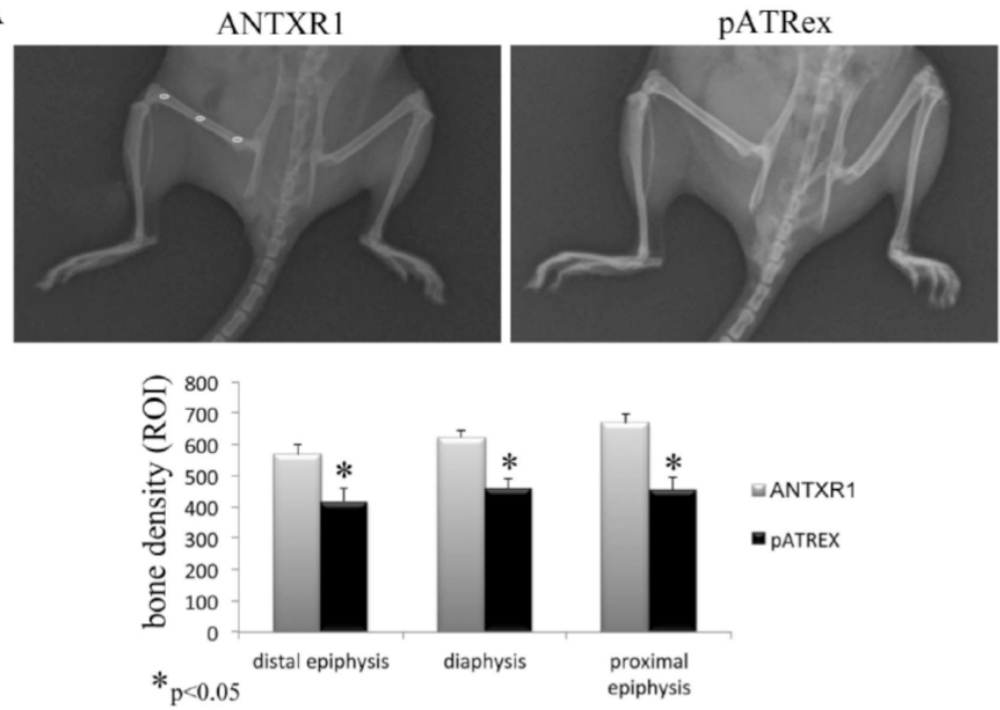

B
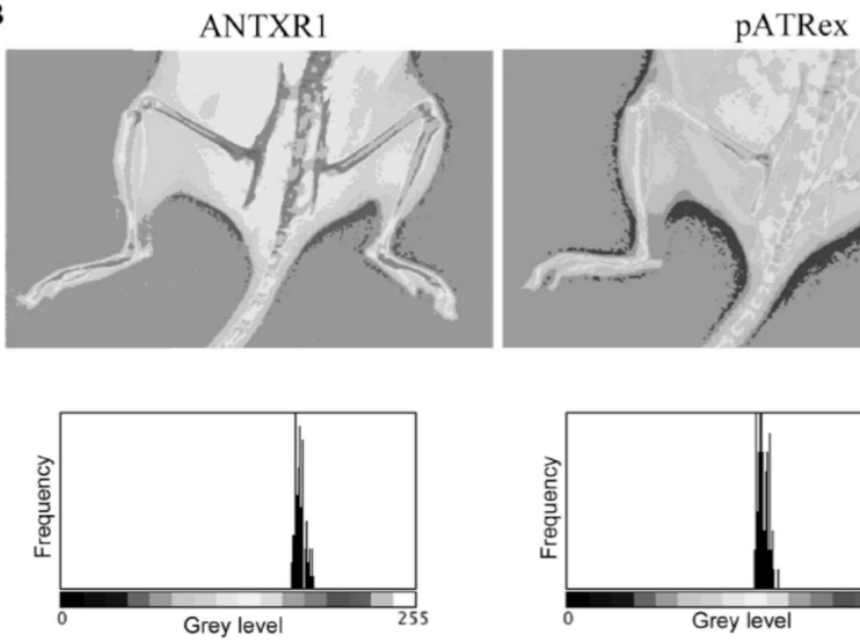

$\mathrm{C}$

collagen I

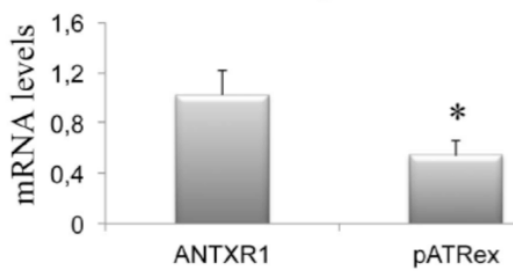

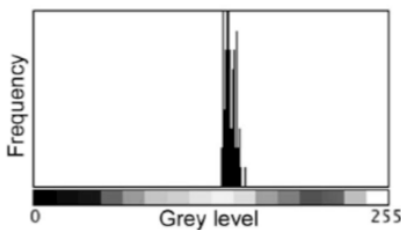

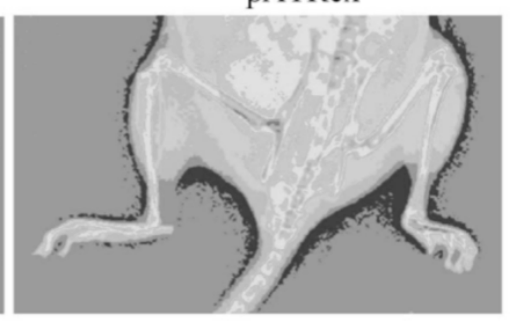

osteocalcin

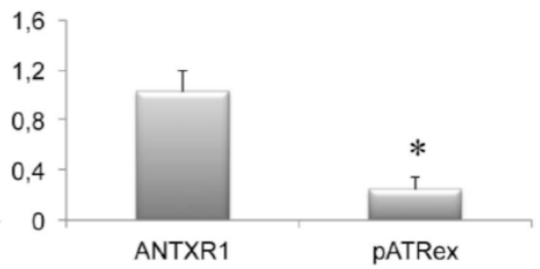

Fig. (3). X-ray images of pelvis and hind limbs of animals of the experiment. Areas of ROI are indicated (small ellipses). Histograms of bone density measured at the levels of distal epiphysis, diaphysis and proximal epiphysis from the original 16 bit DICOM X-ray radiographs (A). Pseudo-color images of pelvis and hind limbs of the same X-ray images, obtained converting to an 8 bit TIFF format from the original 16 bit DICOM X-ray radiographs and pseudocoluring the resultant image using a 16 color look-up table. Histogram of grey levels frequency within subchondral bone ROI (B). N = 7 per group. Gene expression of collagen I and osteocalcin by Real-Time PCR from whole bone marrow (C).

The pathological (resorptive) scenario induced by pATRex injections can be rationally traced back to an increased OCs activity, coupled with an impaired OBs differentiation. Indeed, an increased OCs activity can be documented by a remarkable increase of tartrate resistant acid phosphatase (TRAP) staining around the trabeculae (Fig. 4 A-D) [36]. On the other hand, an impaired OBs differentiation in pATRextreated mice is highlighted by the presence of fat cells (adi- pocytes) within the bone marrow cavity, at the submetaphyseal region (Fig. 4 E-H).

It is well established that fatty infiltrations characterize the process of bone loss due to an impaired mesenchymal stem cells (MSCs) differentiation, which results in decreases maturation of OBs in favor of an increased adipocytes differentiation [37,38]. Coherently with an elevated adipogenic 


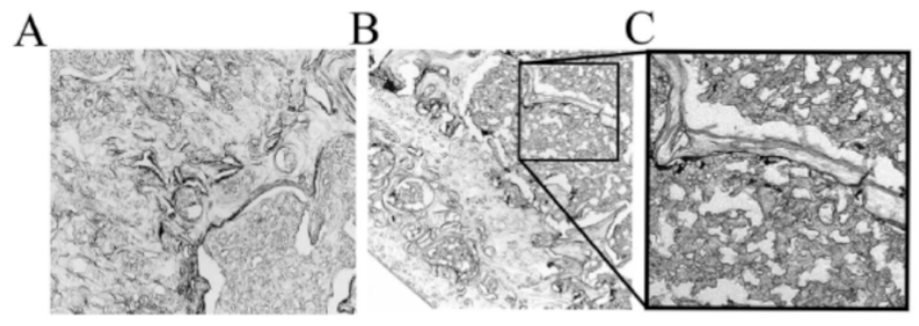

$\mathrm{D}$
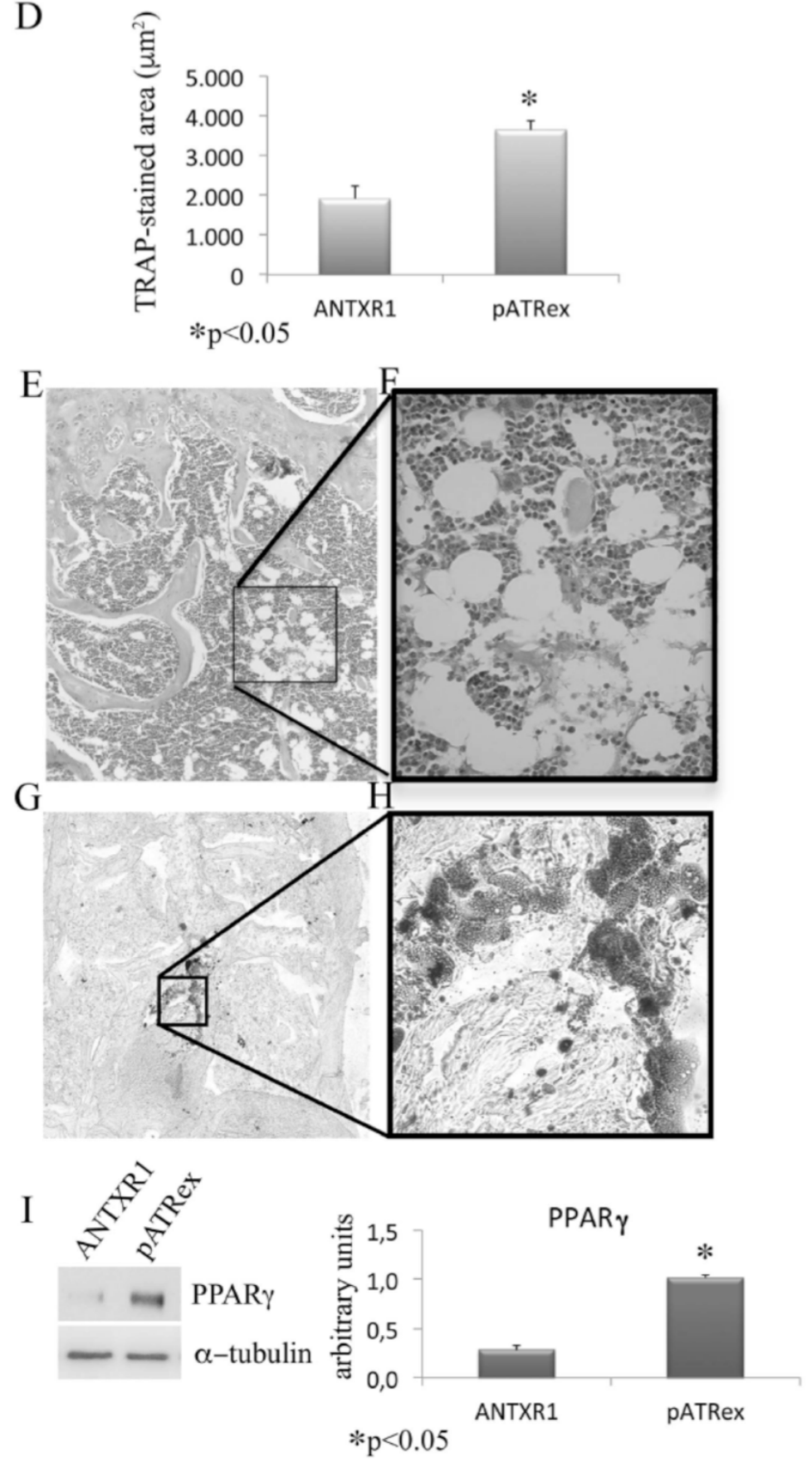

Fig. (4). Epiphyseal regions of distal femurs from ANTXR1 DNA (A) and pATRex DNA-injected mice stained with TRAP (B, C). Magnifications: 20x (A, B); 40x (C). Quantification of TRAP-positive stained area under growth plate of proximal femur per field $\left(0.3 \times 0.3 \mathrm{~mm}^{2}\right)$. Representative reconstruction of metaphyseal regions of distal femurs from pATRex DNA-injected mice stained with hematoxylin-eosin (E, F), emphasizing areas enriched in adypocites. Oil red staining $(\mathbf{G}, \mathbf{H})$. Magnifications: 20x $(\mathbf{E}-\mathbf{H})$. $\mathrm{N}=6$ per group. (I) Representative western blotting of PPAR $\gamma$ from total bone marrow cells obtained from ANTXR1 DNA and pATRex DNA-injected mice (I). Statistical analysis, deriving from three independent experiments, shows increased PPAR $\gamma$ synthesis in bone marrow cells from pATRex DNA-injected mice; $* p<0.05 . \mathrm{N}=6$ per group. 
potential of MSCs in pATRex treated bone, we recorded a strong up-regulation of a master moderator of adipogenesis, such as peroxisome-proliferator-activated receptor- $\gamma$ (PPAR$\gamma$ ) transcription factor (Fig. 4 I) $[39,40]$.

Fully consistent with the morphological studies, were the results obtained by the analysis of the expression of biochemical markers of the bone turnover in bone marrowderived stromal cells (BMSCs). Classically, Receptor Activator of NF- $\kappa B$ ligand (RANKL) and TNF- $\alpha$ are master inflammatory cytokines, that by enhancing the expression of $\mathrm{NF}-\mathrm{kB}$, regulate bone homeostasis by the concomitant stimulation of osteoclastogenesis and inhibition of osteoblast function [41]. It has also been reported that RANKL can induce the differentiation of monocytes and macrophages to preosteoclasts [42]. On the other hand, TNF- $\alpha$ its well known to inhibit osteoblast differentiation on multiple levels $[43,44]$ and its overexpression drives to significant focal and systemic bone erosions. Accordingly, BMCs from pATRexinjected mice showed a marked up-regulation of both TNF $\alpha$ and NF-kb (Fig. 5A). In addition, we also observed increased levels of RANKL expression, while osteoprotegerin (OPG), the soluble (decoy) receptor that inhibits RANKL activity [45] was not affected (Fig. 5B). Moreover, (in line with data in Fig. 3C) Runx-2 and Osterix, key transcription factors for osteoblast differentiation [46, 47], were decreased by $\mathrm{pA}$ TRex treatment (Fig. 5B).
Although we have not direct evidence of the deposition of pATRex aggregates in the osteoporotic bone, future studies will address this issue by immunofluorescent /confocal laser microscopy of EYFP-conjugated pATRex injected plasmid [7]. Moreover, since plasmids biodistribution might change with the administration routes, studies are also planned to explore the osteoporotic potential of pATRex following gene-gun delivery and /or intraperitoneal injection. In the latter case the inoculum introduced into the body cavity, instead of forming a localized deposit, it may spread out and induce disseminated reactions.

\section{CONCLUDING REMARKS}

In summary, the evidence presented here points to a previously unrecognized "hidden" reactogenicity (bone erosion) of pATRex-derived aggregates. Thus, while the study casts doubts about the clinical use of pATRex as vaccine adjuvant, it reminds us that great caution should be exercised in respect to adjuvants postulated to work via cellular toxicity and cell death. Our results provide experimental support to the hypothesis that highly stable protein aggregates in neurodegenerative proteinopathies and other aggregosome disorders, are recognized as dangerous "non self" particles that chronically activate the innate immunosystem. Whether the injection of plasmids coding known pathological aggregateinducing sequences (e.g. A $\beta 42$ and mutant huntingtin polypeptides) induces comparable osteo-immunotoxicty remains

A
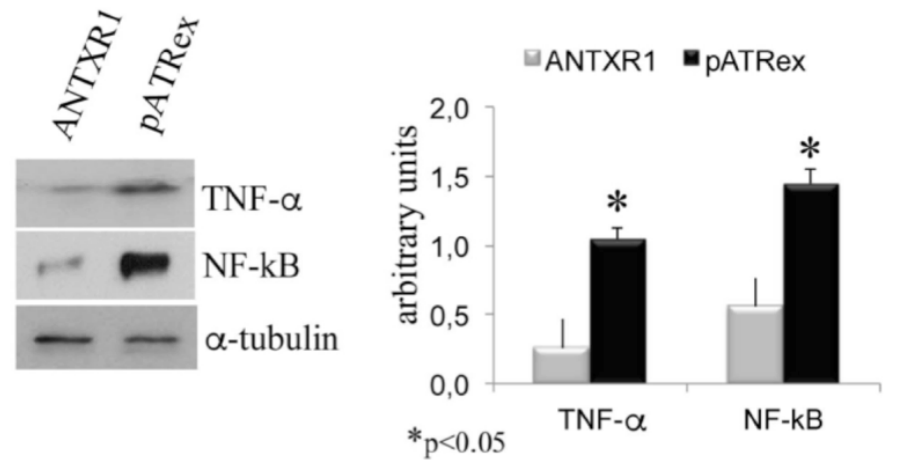

B
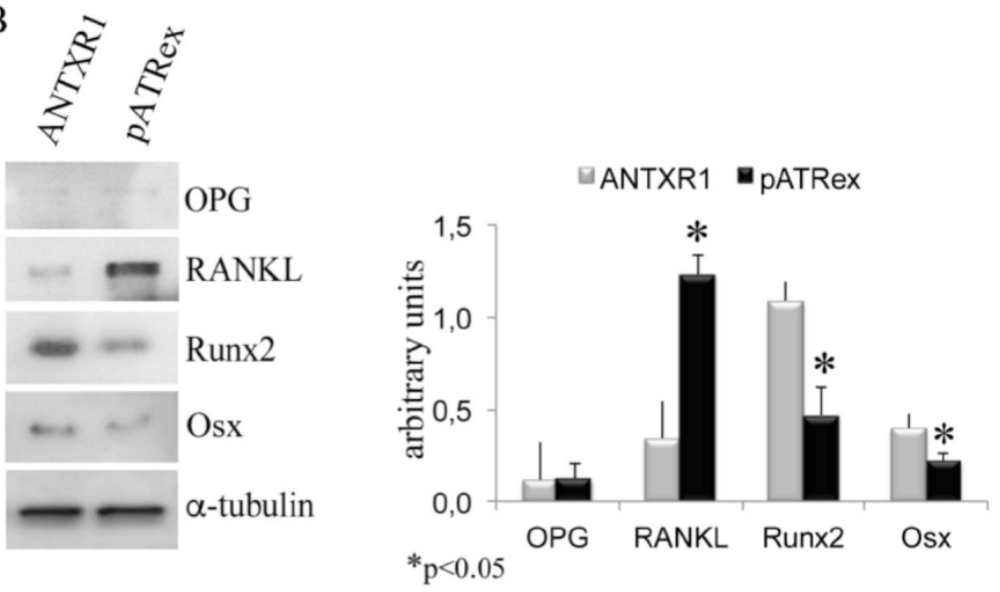

Fig. (5). BMCs were obtained from ANTXR1 DNA and pATRex DNA-injected mice. Note the statistically significant increase of TNF $\alpha$ and NFkB (A) as well as RANKL protein levels after pATRex DNA treatment, over unchanged OPG, and decreased Runx-2 and OSX transcription levels $(\mathbf{B}) .{ }^{*} p<0.05 . \mathrm{N}=7$ per group. 
to be seen. Lastly, beyond this cautionary note, and open questions about the clinical relevance of inflammatory aggregates, we propose pATRex-injected mice as an additional, simplified, mouse model of osteoporosis [48] for the evaluation of mayor pharmaceuticals and novel biodrug candidates.

\section{CONFLICT OF INTEREST}

The authors confirm that this article content has no conflict of interest.

\section{ACKNOWLEDGEMENTS}

This work was partially supported by Strategic University Research Projects (Unicam-FAR) grant to FMV.

\section{REFERENCES}

[1] Donnelly JJ, Ulmer JB, Shiver JW, Liu MA. DNA vaccines. Ann Rev Immunol 1997; 15: 617-48.

[2] Apostolopoulos V, Weiner DB. Development of more efficient and effective DNA vaccines. Exp Rev Vaccine 2009; 8: 1133-4.

[3] Petrovsky N. Freeing vaccine adjuvants from dangerous immunological dogma. Expert Rev Vaccines 2008; 7: 7-10.

[4] Ilyinskii PO, Thoidis G, Sherman MY, Shneider A. Adjuvant potential of aggregate-forming polyglutamine domains. Vaccine 2008; $26: 3223-6$

[5] Fu X, Tao L, Zhang X. A short polypeptide from the herpes simplex virus type 2 ICP10 gene can induce antigen aggregation and autophagosomal degradation for enhanced immune presentation. Hum Gene Ther 2010; 21: 1687-96.

[6] Felicetti P, Mennecozzi M, Barucca A et al. Tumor endothelial marker 8 enhances tumor immunity in conjunction with immunization against differentiation Ag. Cytotherapy 2007; 9: 23-34.

[7] Capitani M, Saade F, Havas KM et al. Plasmids encoding protein aggregation domains act as molecular adjuvants for DNA vaccines. Curr Gene Ther 2014; 14: 161-69.

[8] Pierre SR, Vernace V, Wang Z, Figueiredo-Pereira ME. Assembly of protein aggregates in neurodegeneration: mechanisms linking the ubiquitin/proteasome pathway and chaperones. Madame Curie Bioscience Database [Internet]. Austin (TX): Landes Bioscience; 2000.

[9] Shin JN, Fattah EA, Bhattacharya A, Ko S, Eissa NT. Inflammasome activation by altered proteostasis. J Biol Chem 2013; 288: 35886-95.

[10] Salminen A, Ojala J, Kauppinen A, et al. Inflammation in Alzheimer's disease: amyloid- $\beta$ oligomers trigger innate immunity defence via pattern recognition receptors. Prog Neurobiol 2009; 87: 181-94.

[11] Akiyama $\mathrm{H}$, Barger S, Barnum $\mathrm{S}$ et al. Inflammation and Alzheimer's disease. Neurobiol Aging 2000; 21: 383-421.

[12] McGeer PL, McGeer EG. Innate immunity, local inflammation, and degenerative disease. Sci Aging Knowledge Environ 2002; 2002(29): re3

[13] Straub R. The origin of chronic inflammatory systemic diseases and their sequelae. Academic press; 2015.

[14] Hermesh T, Moltedo B, Moran TM, López CB. Antiviral instruction of bone marrow leukocytes during respiratory viral infections. Cell Host Microbe 2010; 7: 343-53.

[15] Agas D, Marchetti L, Douni E, Sabbieti MG. The unbearable lightness of bone marrow homeostasis. Cytokine Growth Factor Rev 2015; 26: 347-59.

[16] Raggatt LJ, Partridge NC. Cellular and molecular mechanisms of bone remodeling. J Biol Chem 2010; 285: 25103-08.

[17] Redlich K, Smolen JS. Inflammatory bone loss: pathogenesis and therapeutic intervention. Nat Rev Drug Discov 2012; 11: 234-50.

[18] Torsney KM, Noyce AJ, Doherty KM, et al. Bone health in Parkinson's disease: a systematic review and meta-analysis. J Neurol Neurosurg Psychiatry 2014; 85: 1159-66.

[19] Kim YE, Lee WW, Yun JY, et al. Musculoskeletal problems in Parkinson's disease: neglected issues. Parkinsonism Relat Disord 2013; 19: 666-9.
[20] van den Bos F, Speelman AD, Samson M, et al. Parkinson's disease and osteoporosis. Age Ageing 2013; 42: 156-62.

[21] van der Burg JM, Björkqvist M, Brundin P. Beyond the brain widespread pathology in Huntington's disease. Lancet Neurol 2009; 8: 765-74.

[22] Bonelli CR. Bone density and bone turnover in Hungtinton's disease. Osteoporosis Int 2002; 13S: 64.

[23] Otti DV. Osteoporosis in Hungtinton's disease. Neurodegenerative Dis 2007; 4S: 304

[24] Woodman I. Osteoporosis: linking osteoporosis with Alzheimer disease. Nat Rev Rheumatol 2013; 9: 638.

[25] Zhou R, Deng J, Zhang M, Zhou HD, et al. Association between bone mineral density and risk of Alzheimer's disease. J Alzheimers Dis 2011; 24: 101-8.

[26] Li S, Liu B, Zhang L, Rong L. Amyloid $\beta$ peptide is elevated in osteoporotic bone tissues and enhances osteoclast function. Bone 2014; 61: 164-75.

[27] Coelho-Castelo AM, Trombone AP, Rosada RS, et al. Tissue distribution of a plasmid DNA encoding Hsp65 gene is dependent on the dose administered through intramuscular delivery. Genetic Vaccines and Therapy 2006; $4: 1-10$.

[28] Rush CM, Mitchell TJ, Garside P. A detailed characterisation of the distribution and presentation of DNA vaccine encoded antigen. Vaccine 2010; 28: 1620-34.

[29] Sabbieti MG, Agas D, Capitani M, et al. Plasmid DNA-coding p62 as a bone effective anti inflammatory /anabolic agent. Oncotarget 2015; 6: 3590-99.

[30] Egan KP, Brennan TA, Pignolo RJ. Bone histomorphometry using free and commonly available software. Histopathology 2012; 61 1168-73.

[31] Shimizu H, Nakagami H, Yasumasa N, et al. Cilnidipine, but not amlodipine, ameliorates osteoporosis in ovariectomized hypertensive rats through inhibition of the N-type calcium channel. Hypertens Res 2012; 35: 77-81.

[32] McManus MM, Grill RJ. Longitudinal evaluation of mouse hind limb bone loss after spinal cord injury using novel, in vivo, methodology. J Vis Exp 2011; 7: 58.

[33] Waung JA, Maynard SA, Gopal S, et al. Quantitative X-ray microradiography for high-throughput phenotyping of osteoarthritis in mice. Osteoarthritis Cartilage 2014; 22: 1396-400.

[34] Sabbieti MG, Agas D, Marchetti L, et al. BMP-2 differentially modulates FGF-2 isoform effects in osteoblasts from newborn transgenic mice. Endocrinology 2013; 154: 2723-33.

[35] Gao Y, Grassi F, Ryan MR, et al. IFN- $\gamma$ stimulates osteoclast formation and bone loss in vivo via antigen-driven T cell activation. J Clin Invest 2007; 117: 122-32.

[36] Kirstein B, Chambers TJ, Fuller K. Secretion of tartrate-resistant acid phosphatase by osteoclasts correlates with resorptive behavior J Cell Biochem 2006; 98: 1085-94.

[37] Rodriguez JP, Garat S, Gajardo, et al. Abnormal osteogenesis in osteoporotic patients is reflected by altered mesenchymal stem cells dynamics. J Cell Biochem 1999; 75: 414-23.

[38] Verma S, Rajaratnam JH, Denton J, et al. Adipocytic proportion of bone marrow is inversely related to bone formation in osteoporosis. J Clin Pathol 2002; 55: 693-8.

[39] Rozman C, Feliu E, Berga L, et al. Age-related variations of fat tissue fraction in normal human bone marrow depend both on size and number of adipocytes:a stereological study. Exp Hematol 1989; $17: 34-7$

[40] Akune T, Ohaba S, Kamekura S, et al. PPARgamma insufficiency enhances osteogenesis through osteoblast formation from bone marrow progenitors. J Clin Invest 2004; 113: 846-55.

[41] Krum SA, Chang J, Miranda-Carboni G, Wang CY. Novel functions for NFkB: inhibition of bone formation. Nat Rev Rheumato 2010; 6: 607-11.

[42] Teitelbaum SL. Bone resorption by osteoclasts. Science 2000; 289: 1504-08.

[43] Zaidi M. Skeletal remodeling in health and disease. Nat Med 2007; 13: 791-801

[44] Redlich K, Smolen JS. Inflammatory bone loss: pathogenesis and therapeutic intervention. Nat Rev Drug Discov 2012; 11: 234-50.

[45] Lacey DL, Timms E, Tan HL, et al. Osteoprotegerin ligand is a cytokine that regulates osteoclast differentiation and activation. Cell 1998; 93: 165-76. 
[46] Otto F, Thornell A, Crompton T, et al. Cbfa1, a candidate gene for cleidocranial dysplasia syndrome, is essential for osteoblast differentiation and bone development. Cell 1997; 89: 765-71.

[47] Yang X, Karsenty G. Transcription factors in bone: developmental and pathological aspects. Trends Mol Med 2002; 8: 340-45.
[48] Golde TE, Miller VM. Proteinopathy-induced neuronal senescence: a hypothesis for brain failure in Alzheimer's and other neurodegenerative diseases. Alzheimers Res Ther 2009; 1: 5. 